EPSC Abstracts

Vol. 15, EPSC2021-267, 2021, updated on 05 Aug 2021

https://doi.org/10.5194/epsc2021-267

European Planetary Science Congress 2021

(c) Author(s) 2021. This work is distributed under

the Creative Commons Attribution 4.0 License.

\title{
Planetary VO services on VESPA : MCD, SPICAM and EXOTOPO
}

Chloé Azria ${ }^{1}$, Anni Määttänen ${ }^{2}$, Ehouarn Millour ${ }^{3}$, Frédéric Schmidt ${ }^{4}$, François Andrieu ${ }^{4}$, Erard Stéphane ${ }^{5}$, Cecconi Baptiste ${ }^{5}$, and Pierre Le Sidaner ${ }^{5}$

${ }^{1}$ IPSL, Paris, France (chloe.azria@universite-paris-saclay.fr)

${ }^{2}$ LATMOS/IPSL, Sorbonne université, UVSQ Université Paris-Saclay, CNRS, Paris, France

${ }^{3} \mathrm{LMD} / \mathrm{IPSL}$, Paris, France

${ }^{4}$ GEOPS/IPSL, Université Paris Saclay, Orsay, France

${ }^{5}$ LESIA/Observatoire de Paris, Paris/Meudon, France

\section{Introduction}

The development of VESPA in the Europlanet 2024 program encompasses the improvement of Virtual Observatory (VO) services to enlarge and update its content.

VESPA services use the EPN-TAP protocol defined for Planetary Science and Heliophysics. As a restriction of the more general TAP protocol, such services benefit from TAP-compliant tools and protocols previously defined in the VO [1].

The development of three Planetary services will be described here. The creation of a service providing outputs of a model of topography of exoplanets called EXOTOPO, and the update of two services providing profiles of various parameters of Mars atmosphere : SPICAM data and MCD simulations. These services are provided with user manuals linked in their description to explain the main search criteria that can be used. The three services are implemented on DaCHS, a commonly used TAP server provided by Heidelberg University.

\section{EXOTOPO Service}

Dataset:

The new service EXOTOPO gives access to simulation results based on a statistical model of topographies of (exo)planet surfaces (3D visualisator : [2]). The model is based on 3 parameters ( $\mathrm{H}$ : degree of smoothness, C1 : degree of intermittency, alpha : degree of multifractality), see Landais et. al 2018 [3] for more information. A new data set was generated to provide a larger range of parameter variation [4]. For each combination of parameters ( $\mathrm{H}, \mathrm{C} 1$, alpha and the Random Seed identifier), 5 types of data outputs are provided (see Fig. 1): An elevation map ; An earth-like colorized texture with continents and oceans, with and without hill-shading ; A gray texture map, small body-like, with and without hill-shading ; additionally, two spherical plots of altitude in 3D with the hill-shaded textures are provided as thumbnails of elevation maps. 

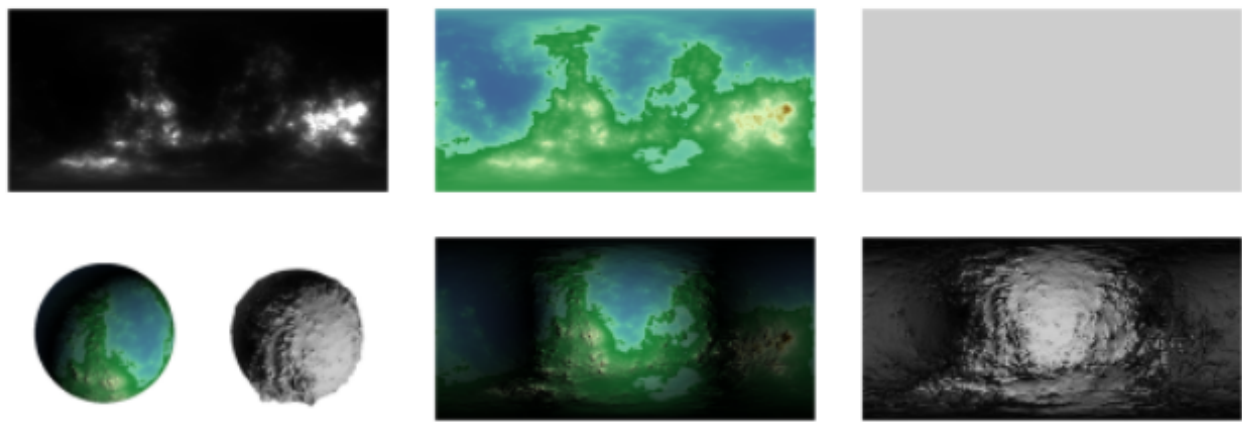

Figure 1: Datasets included in the EXOTOPO service. From Top-right to Bottom-left: Synthetic topography; Color texture of the synthetic topography : Earth-like coloration; B/W texture of the synthetic topography : Gray uniform coloration ; 3D rendering of the shape model; Color texture of the synthetic topography, including shadows; B/W texture of the synthetic topography, including shadows

\section{Implementation :}

The server hosting DaCHS is deployed on Docker from a VM (Virtual Machine) in ESPRI data center. A reverse-proxy on ESPRI redirects the server address to https://dachs-vo.ipsl.fr for outside. Data files are directly uploaded by ESPRI and linked in the service. The topographic map is provided in fits format with geological referencement and texture maps are provided in png, both are readable by vo tools like Aladin.

\section{MCD and SPICAM services}

\section{Datasets :}

The MCD (Mars Climate Database [5]) is a database of Mars atmospheric parameters based on a modelization of Mars atmosphere developed in LMD (Laboratoire de Météorologie Dynamique). The database provides various quantities (pressure, temperature, wind, radiative fluxes, composition, ...) for a list of scenarios of dust and storm and for Martian years 24 to 32 (see the user manual [6] for more information). The MCD can be accessed through a web interface, or a full version can be downloaded and interrogated by a Fortran function. The VO service implemented in VESPA provides an alternative access to the MCD which allows the selection of data subsets using various research criteria.

SPICAM (Spectroscopy for Investigation of Characteristics of the Atmosphere of Mars [7]) is an ultra-violet [118-300]nm and infrared [1.0-1.7] $\mathrm{mm}$ spectrometer on board Mars Express. It has been observing the Martian atmosphere from January 2004 (Mars Year 27) onwards with nadir, limb and stellar/solar occultation measurements. The UV channel ceased operations in 2014, but the IR channel is still currently collecting data. Four types of data profiles derived from occultations in the UV channel are provided in the VO service SPICAM : aerosol extinction [8] derived from solar occultations, temperature, and densities of $\mathrm{CO} 2$ [9] and $\mathrm{O} 3$ [10] from stellar occultations. On the VO service, each SPICAM profile is associated with an MCD profile at the same coordinates for the current Mars Year scenario on the service (see Fig. 2). Furthermore, datalinks for each granule link to associated MCD profiles, with the possibility to select any available scenario. 


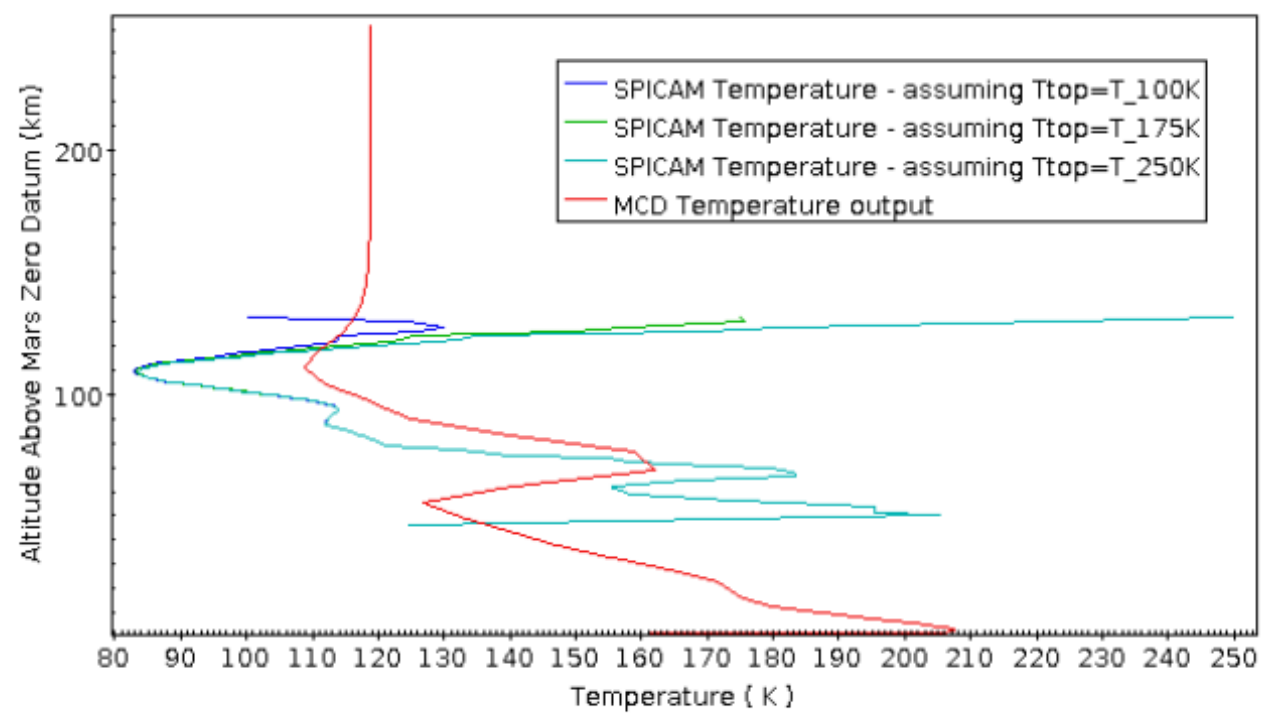

Figure 2 : Comparison (plotted on the VO tool Topcat) of temperature profiles from SPICAM measurements (assuming that the temperature at $130 \mathrm{~km}$ is $100 \mathrm{~K}, 175 \mathrm{~K}$ and $250 \mathrm{~K}$ ) for orbit 2888A1 and MCD simulations at the same coordinates, with the corresponding Mars Year scenario (MY28).

Implementation :

The servers for MCD and SPICAM services are administered directly at the LMD and LATMOS laboratories, on VM that runs on debian 10 and hosting gavodachs2, and Apache configured for cgi (Common Gateway Interface). Data from SPICAM measurements are directly uploaded using Apache whereas MCD profiles are computed on-the-fly using cgi-scripts in python to call mcd and generate queried profiles. Both data from MCD and SPICAM are provided in VOtable format [11] which can be handled by VO tools such as TOPCAT and be clearly referenced.

\section{Conclusion :}

The Virtual Observatory (VO) addresses the issue of providing FAIR (findable, accessible, interoperable, reusable) access to scientific data. VESPA services apply this paradigm to resources in Planetary Science and Heliophysics, with uniformed metadata, a powerful query interface to select data, and specialized formats for various data types. Furthermore, the VO provides the SAMP interface, a very simple way to plot and compare data that can be obtained from various services.

The Europlanet-2024 Research Infrastructure project has received funding from the European Union's Horizon 2020 research and innovation programme under grant agreement No 871149.

\section{References:}

[1] Erard et al , VESPA: A community-driven Virtual Observatory in Planetary Science, Planetary and Space Science, Volume 150, January 2018, Pages 65-85 10.1016/j.pss.2017.05.013 https://doi.org/10.1016/j.pss.2017.05.013

[2] 3D visualisator of some Exotopo model outputs https://data.ipsl.fr/exotopo/

[3] F Landais, F Schmidt, S Lovejoy, Topography of (exo)planets, Monthly Notices of the Royal Astronomical Society, Volume 484, Issue 1, March 2019, Pages 787-793, https://doi.org/10.1093/mnras/sty3253 
[4] EXOTOPO data set (former version http://dx.doi.org/10.14768/20181024001.1; new version https://data.ipsl.fr/multifractal-topography/ )

[5] Mars Climate Database : Forget et al. 1999, Improved general circulation models of the Martian atmosphere from the surface to above $80 \mathrm{~km}$; Millour et al. 2018, The Mars Climate Database (Version 5.3) .

[6] Mars Climate Database parameters v5.3 user manual http://wwwmars.Imd.jussieu.fr/mars/info_web/user_manual_5.3.pdf

[7] SPICAM instrument https://mars-express.cnes.fr/en/MEX/spicam.htm

[8] Määttänen et al. (2013), A complete climatology of the aerosol vertical distribution on Mars from MEx/SPICAM UV solar occultations, Icarus, 223, doi:10.1016/j.icarus.2012.12.001

[9] Forget et al. (2009), Density and temperatures of the upper Martian atmosphere measured by stellar occultations with Mars Express SPICAM, J. Geophys. Res., 114, E01004, doi:10.1029/2008JE003086

[10] Lebonnois et al. (2006), Vertical distribution of ozone on Mars as measured by SPICAM/Mars Express using stellar occultations, J. Geophys. Res., 111, E09S05, doi:10.1029/2005JE002643

[11] VOtable format standards (v 1.4) https://www.ivoa.net/documents/VOTable/20191021/RECVOTable-1.4-20191021.pdf 